Supporting Information

\title{
Tailored Reconstituted Lipoprotein for Site-Specific and Mitochondria-Targeted Cyclosporine A Delivery to Treat Traumatic Brain Injury
}

Lepei Chen ${ }^{\dagger, \perp}$, Qingxiang Song ${ }^{\dagger, \perp}$, Yaoxing Chen ${ }^{\dagger}$, Shuang Mengl, Mengna Zheng ${ }^{\dagger}$, Jialin Huang ${ }^{\dagger, \neq}$, Qian Zhang ${ }^{\dagger}$, Jiyao Jiang ${ }^{\ddagger}$, Junfeng Feng ${ }^{\ddagger}$, Hongzhuan Chen ${ }^{\dagger, \S}$, Gan Jiang ${ }^{*, \dagger}$, Xiaoling $G a o^{*, \dagger}$

† Department of Pharmacology and Chemical Biology, State Key Laboratory of Oncogenes and Related Genes, Shanghai Universities Collaborative Innovation Center for Translational Medicine, Shanghai Jiao Tong University School of Medicine, 280 South Chongqing Road, Shanghai 200025, China.

‡ Department of Neurological Surgery, Renji Hospital, School of Medicine, Shanghai Jiao Tong University, 1630 Dongfang Road, Shanghai 200127, China.

$\S$ Institute of Interdisciplinary Integrative Biomedical Research, Shuguang Hospital, Shanghai University of Traditional Chinese Medicine, 1200 Cailun Road, Shanghai 201210, China.

|| Core facility of basic medical sciences, Shanghai Jiao Tong University School of Medicine, 280 South Chongqing Road, Shanghai 200025, China. 
* Corresponding author Email: scmcyidingkaixin@163.com (G.J.), shellygao1@sjtu.edu.cn (X.L.G) $\perp$ Authors contributed equally to this work.

Table S1. Particle size, Zeta potential and PDI of nanoparticles

\begin{tabular}{llll}
\hline & Particle size $\mathbf{( n m )}$ & \multicolumn{1}{l}{ Zeta potential } & Polydispersity \\
& & $\mathbf{( m V )}$ & index \\
\hline RL & $27.51 \pm 4.8$ & $-28.91 \pm 1.47$ & 0.23 \\
MCRL & $36.77 \pm 5.0$ & $-32.68 \pm 4.87$ & 0.27 \\
CsA-MCRL & $66.5 \pm 7.68$ & $-36.18 \pm 3.41$ & 0.23 \\
DiR-labeled RL & $39.88 \pm 1.19$ & $-28.73 \pm 0.64$ & 0.35 \\
DiR-labeled MCRL & $47.57 \pm 5.16$ & $-24.5 \pm 2.11$ & 0.21 \\
DiI-labeled RL & $32.95 \pm 2.5$ & $-32.23 \pm 1.8$ & 0.38 \\
DiI-labeled MCRL & $38.69 \pm 4.72$ & $-36.15 \pm 1.08$ & 0.21 \\
\hline DiI- CsA-MCRL & $69.5 \pm 6.68$ & $-30.18 \pm 2.51$ & 0.31 \\
\hline
\end{tabular}


A

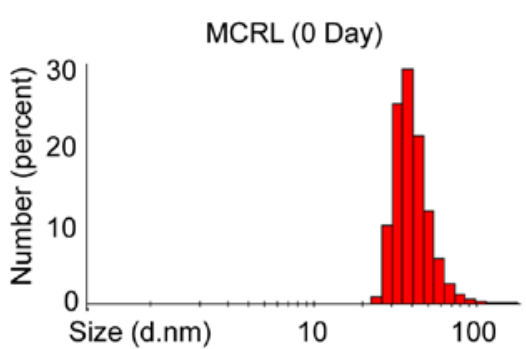

B

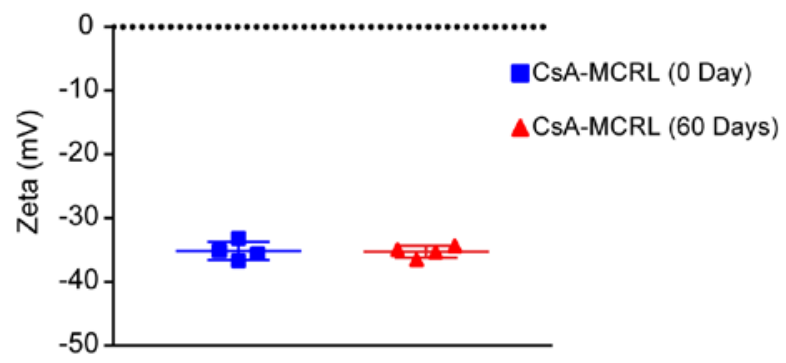

CsA-MCRL (0 Day)

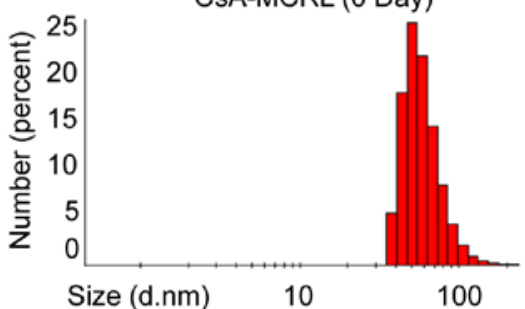

C

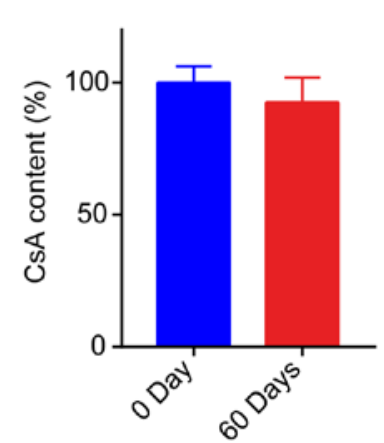

CsA-MCRL (60 Days)

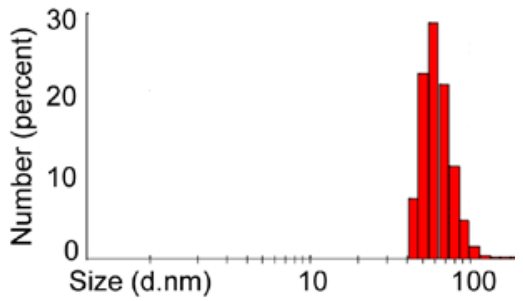

D

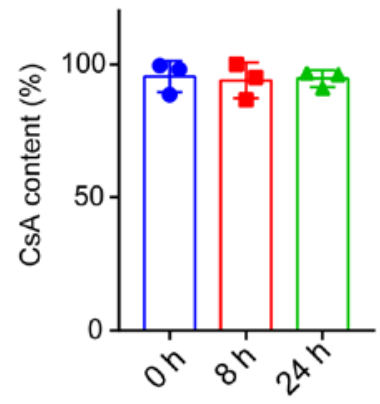

Figure S1. Stability of CsA-MCRL in storage and in serum. (A) The particle size distribution of MCRL and CsA-MCRL at 0 day and 60 days after synthesis under a dynamic light scattering. (B) The zeta potential of CsA-MCRL at 0 day and 60 days after synthesis under a dynamic light scattering. (C) CsA content in CsA-MCRL at 0 day and 60 days after storage at $4^{\circ} \mathrm{C}$. The CsA concentration was quantified by liquid chromatography mass spectrometry analysis. The content of CsA in CsA-MCRL at 0 day was set as 100\%. (D) CsA content in CsA-MCRL after incubation in $10 \%$ serum. The CsA concentration was quantified by liquid chromatography mass spectrometry analysis. The content of CsA in CsA-MCRL at $0 \mathrm{~h}$ was set as $100 \%$. For (B), (C) and (D), Unpaired student's t-test (two-tailed) was used for comparison between two groups (n.s.). 


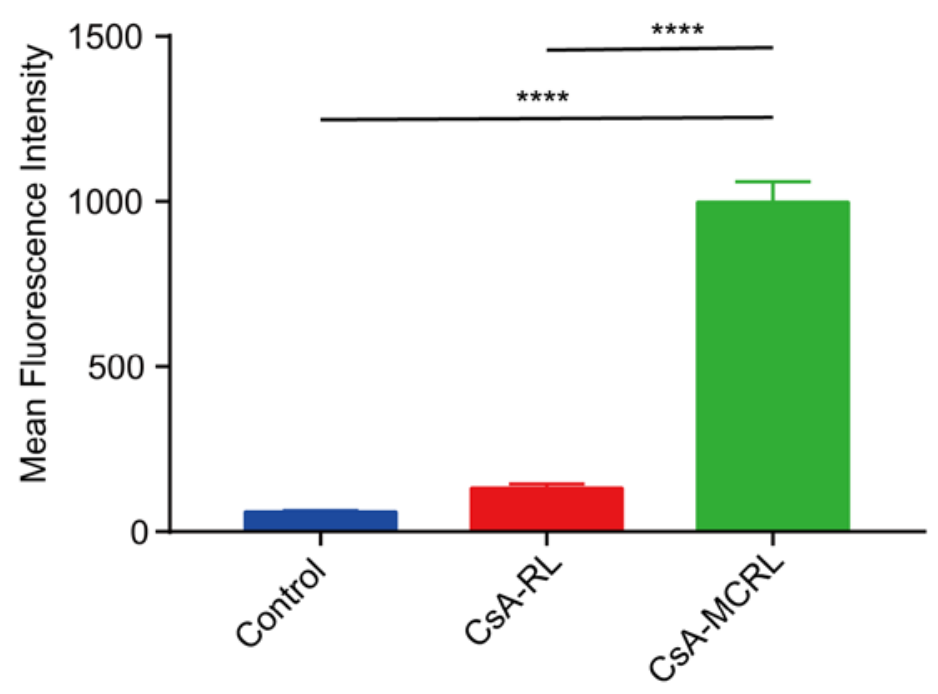

Figure S2. The internalization levels of CsA-RL and CsA-MCRL into the primary neurons by high content screening analysis. The significance of the differences was evaluated by one-way ANOVA followed by Bonferroni test. $(* * * * p<0.0001)$

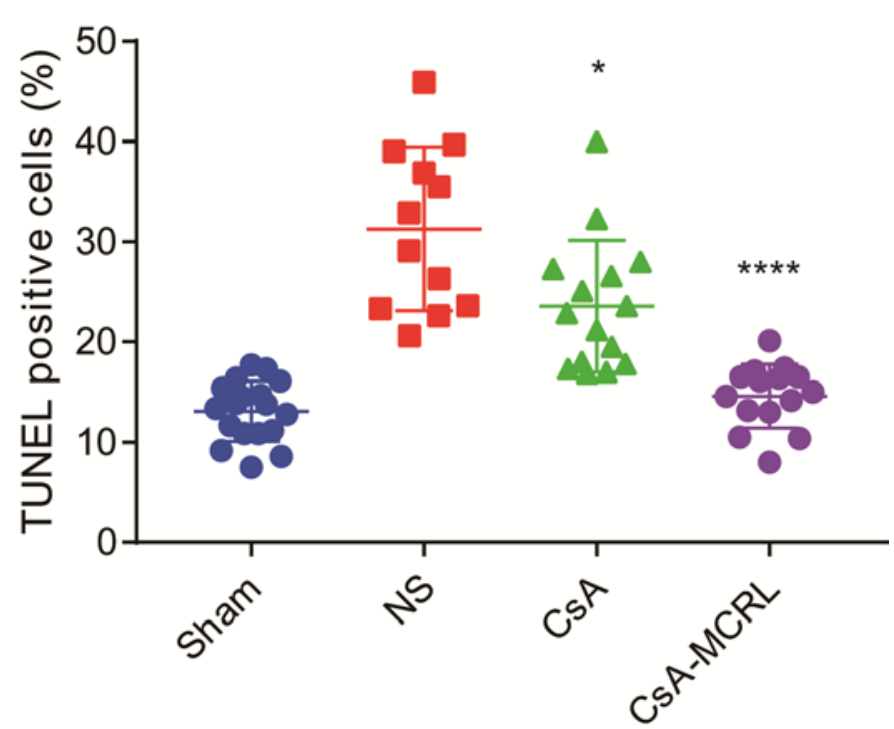

Figure S3. CsA-MCRL rescued severe apoptosis at the TBI sites in mild CCI mice model. ( $\mathrm{n}=3$, Region of interest=12-18) The significance of the differences was evaluated by one-way ANOVA followed by Bonferroni test. $\left({ }^{*} \mathrm{p}<0.05,{ }^{* * * *} \mathrm{p}<0.0001\right)$ 

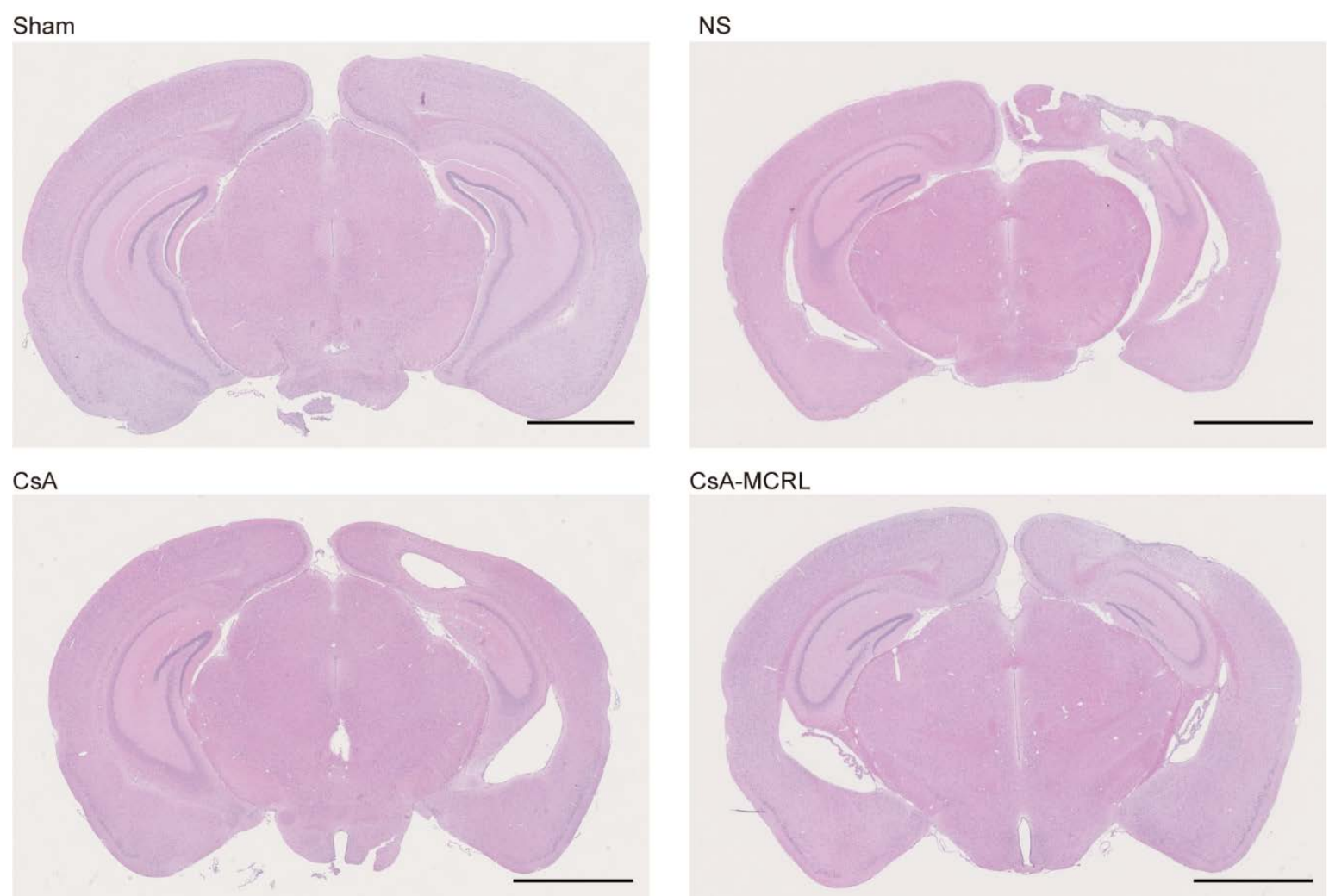

Figure S4. The structure of whole brains after daily treatment of saline, CsA (20 mg kg-1 $\left.\mathrm{d}^{-1}\right)$ and CsA-MCRL (1.26 mg kg $\left.{ }^{-1} \mathrm{~d}^{-1}\right)$ for two weeks. Bar, $1 \mathrm{~mm}$.
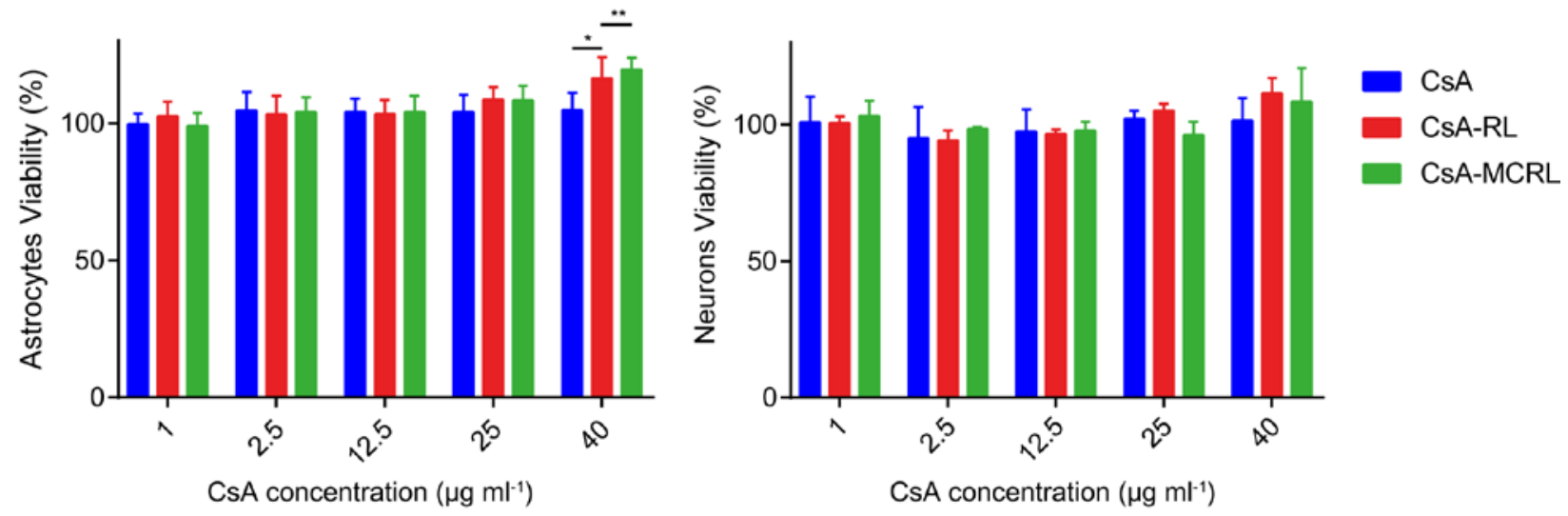

Figure S5. Cell viability of the primary astrocytes and neurons after the treatment with CsA, CsA-RL and CsA-MCRL. The significance of the differences was evaluated by one-way ANOVA followed by Bonferroni test. $\left({ }^{*} \mathrm{p}<0.05,{ }^{* *} \mathrm{p}<0.01\right)$ 